Clinical symptoms are sudden chest pain, dyspnea, and, less commonly, dysphagia and hoarseness. Physically, subcutaneous air and a typical crunching sound are present.

When chest pain and dyspnea are present, anxiety and panic attack are frequent. Panic disorders are often observed in anorexic patients: when chest pain and/or dyspnea or dysphagia appear, spontaneous PM should be considered. ${ }^{1}$

At present, 20 cases of spontaneous PM have been described in the literature. ${ }^{2-4}$

PM in anorexic patients is sometimes produced by selfinduced vomiting. ${ }^{3}$ Our patient had no history of vomiting, and the thorough diagnostic procedures (thoracic computed tomographic scan, esophageal radiologic evaluation, and laryngopharyngoscopy) showed no signs of esophageal or upper airway laceration.

The pathophysiologic mechanism of PM in our patient can be explained by an alveolar wall rupture, with consequent air leak into the mediastinum. The air was interestingly confined to the mediastinum only: no signs of pneumothorax were evident.

PM is sometimes observed in AN: self-induced vomiting causing an esophageal laceration is frequently the cause of $\mathrm{PM}$ in these patients.

Anorexic patients with severe malnutrition are at high risk for PM or pneumothorax. ${ }^{4}$
Animal studies reveal that calorie restriction results in an important loss of alveoli and a fall in gas-exchange tissue and thinner alveolar walls. ${ }^{5}$ The organism sacrifices the nonessential structures for gluconeogenesis to provide glucose for the brain and proteins for the muscles. Because the oxygen consumption falls during calorie restriction, some lung tissue is sacrificed. With thinner alveolar walls and the loss of alveoli, malnourished anorexic patients are at risk of alveolar wall rupture.

In conclusion, we can suppose that severe malnutrition, causing weakness of the alveolar wall and thinning of the connective tissue, can explain the occurrence of the PM in our patient.

\section{References}

1. Karim A, Ahmed S, Rossoff L. Pneumomediastinum simulating a panic attack in a patient with anorexia nervosa. Int J Eat Dis. 2002;33:104-7.

2. Overby KJ, Litt IF. Mediastinal emphysema in an adolescent with anorexia nervosa and self-induced emesis. Pediatrics. 1988;81:134-6.

3. Folsom T, Realmuto MD. Pneumomediastinum in an adolescent female with anorexia nervosa. Int J Eat Dis. 2006;3:67-70.

4. van Veelen I, Hogerman PHG, van Elburg A, Nielsen-Abbring FW, Heggelman BGF, Mahieu HF. Pneumomediastinum: a rare complication of anorexia nervosa in children and adolescents. A case study and review of the literature. Eur J Pediatr. 2008;167:171-4.

5. Massaro D, Massaro GD, Baras A, Hoffman EP, Clerch LB. Calorie-related rapid onset of alveolar loss, regeneration, and changes in mouse lung gene expression. Am J Physiol Lung Cell Mol Physiol. 2004;286:L896-906.

\title{
Transcranial Doppler ultrasonography: A reliable method of monitoring pulsatile flow during cardiopulmonary bypass in infants and young children
}

\author{
Ashley Rogerson, BS, ${ }^{\mathrm{a}}$ Yulong Guan, MD, ${ }^{\mathrm{a}}$ Stephen J. Kimatian, MD, ${ }^{\mathrm{b}}$ Allen Kunselman, MA, ${ }^{\mathrm{a}}$ \\ J. Brian Clark, MD, ${ }^{\mathrm{a}, \mathrm{b}}$ John L. Myers, MD, ${ }^{\mathrm{a}, \mathrm{b}}$ and Akif Ündar, PhD, ${ }^{\mathrm{a}, \mathrm{c}, \mathrm{d}}$ Hershey, Pa
}

\footnotetext{
From the Pediatric Cardiac Research Laboratories, Departments of Pediatrics ${ }^{\mathrm{a}}$ Pediatric Anesthesiology, ${ }^{\mathrm{b}}$ Surgery, ${ }^{\mathrm{c}}$ and Bioengineering, ${ }^{\mathrm{d}}$ Penn State Milton S. Hershey Medical Center, Penn State College of Medicine, Penn State Hershey Children's Hospital, Hershey, Pa.

Disclosures: None.

This research was supported by the Children's Miracle Network.

Received for publication Dec 23, 2008; revisions received Feb 9, 2009; accepted for publication Feb 16, 2009; available ahead of print June 15, 2009.

Address for reprints: Akif Ündar, PhD, Associate Professor of Pediatrics, Surgery, and Bioengineering, Penn State College of Medicine, Department of Pediatrics-H085, 500 University Dr, PO Box 850, Hershey, PA 17033-0850 (E-mail: aundar@psu. edu).

J Thorac Cardiovasc Surg 2010;139:e80-2

$0022-5223 / \$ 36.00$

Copyright (c) 2010 by The American Association for Thoracic Surgery doi:10.1016/j.jtcvs.2009.02.053
}

With a reported incidence ranging from $2 \%$ to $25 \%$, neurologic morbidity remains a leading complication in infants and children undergoing cardiac operations requiring the use of cardiopulmonary bypass (CPB). ${ }^{1}$ Maintaining optimal cerebral perfusion and hemodynamics during $\mathrm{CPB}$ procedures should be the goal to prevent long-term neurologic complications after pediatric open cardiac operations.

Pulsatile flow during CPB generates significantly more hemodynamic energy levels than does nonpulsatile flow at the same mean arterial pressures and pump flow rates in neonates and infants. ${ }^{2,3}$ Extra energy generated by pulsatile flow may maintain better cerebral blood flow. ${ }^{3}$ We focused on Gosling's pulsatility index (PI) inasmuch as it is accepted as a reliable measure of pulsatility and available on all 
TABLE 1. Perioperative patient demographics and perfusion data

\begin{tabular}{lccc}
\hline & $\begin{array}{c}\text { Nonpulsatile } \\
(\text { mean } \pm \text { SEM) }\end{array}$ & $\begin{array}{c}\text { Pulsatile } \\
(\text { mean } \pm \text { SEM) }\end{array}$ & P values \\
\hline Jenkins & $2.15 \pm 0.15$ & $2.00 \pm 0.20$ & .76 \\
$\quad$ risk category & & & \\
Age (d) & $605.9 \pm 186.1$ & $747.3 \pm 179.0$ & .59 \\
Weight $(\mathrm{kg})$ & $8.92 \pm 1.38$ & $10.77 \pm 1.30$ & .34 \\
BSA $\left(\mathrm{m}^{2}\right)$ & $0.42 \pm 0.05$ & $0.49 \pm 0.05$ & .31 \\
Pump & $117.15 \pm 14.56$ & $115.69 \pm 8.78$ & .93 \\
$\quad$ time (min) & & & \\
Crossclamp & $75.92 \pm 11.88$ & $86.46 \pm 7.35$ & .46 \\
$\quad$ time (min) & & & \\
Temperature & $32.45 \pm 0.50$ & $32.89 \pm 0.52$ & .55 \\
$\left.\quad{ }^{\circ} \mathrm{C}\right)$ & & & \\
MAP before & $67.62 \pm 3.73$ & $59.55 \pm 2.14$ & .09 \\
$\quad$ CPB (mm Hg) & & & \\
MAP after & $65.92 \pm 2.79$ & $78.09 \pm 3.91$ & .02 \\
$\quad$ CPB (mm Hg) & & & \\
Urine output (mL) & $58.69 \pm 16.02$ & $72.23 \pm 15.10$ & .54 \\
PFH before CPB & $9.08 \pm 1.26$ & $7.18 \pm 1.15$ & .30 \\
PFH after CPB & $54.38 \pm 8.91$ & $40.91 \pm 8.14$ & .30
\end{tabular}

$B S A$, Body surface area; $C P B$, cardiopulmonary bypass; $M A P$, mean arterial pressure; $P F H$, plasma free hemoglobin; SEM, standard error of the mean.

transcranial Doppler (TCD) monitoring units. PI is calculated via the following equation ${ }^{4}$ :

$$
\mathrm{PI}=\left(\mathrm{P}_{\mathrm{sys}}-\mathrm{E}_{\mathrm{dia}}\right) / \text { Mean }
$$

where $\mathrm{P}_{\text {sys }}=$ peak systolic flow in the highest velocity; $\mathrm{E}_{\text {dia }}$ $=$ end - diastolic flow is the lowest velocity; and mean $=$ mean velocity.

The goal of this brief communication is to demonstrate that TCD is a feasible method of measuring pulsatility in cerebral vasculature and can be used in future research exploring the neurologic benefits of pulsatile flow in pediatric CPB.

\section{METHODS}

Before the operation, 26 pediatric patients undergoing cardiac surgery with CPB for congenital heart defects from March 2007 to August 2008 were randomly assigned to pulsatile $(n=13)$ and nonpulsatile $(n=13)$ groups. In addition to the institutional review board approval (IRB Protocol No: 19299), Data Safety and Monitoring Board has been established for this project. Analysis of the groups according to demographic and surgical data from the perioperative period is shown in Table 1 .

The CPB circuit was composed of a Jostra HL-20 heart-lung machine, a Jostra-30 heart-cooler system (Jostra USA, Austin, Tex), a Capiox RX 05 Baby-RX hollow-fiber membrane oxygenator, and a Capiox pediatric 32- $\mu \mathrm{m}$ arterial filter (Terumo Corporation, Tokyo, Japan). All components of the circuit are approved by the Food and Drug Administration (FDA) and selected on the basis of previous studies demonstrating high-quality pulsatile perfusion. ${ }^{5}$ Target hematocrit value was $25 \%$ to $27 \%$ and modified ultrafiltration was used in every patient. Pulsatile flow settings were as follows: pump rate of 100 beats/min, $10 \%$ of the base flow, $20 \%$ of the pump head start point, and $80 \%$ of the pump head stop point.

TCD ultrasound sensors (Nicolet Biomedical Inc., Madison, Wis) were placed in the right temporal window to capture the middle cerebral artery (MCA) and on the CPB circuit tubing before the aortic cannula. PI was recorded throughout the procedure using WinTCD (2002) software. The patient's intrin- sic MCA pulsatility was noted before the first incision. Another baseline PI measurement was recorded from both the CPB tubing and the MCA while the patient was supported by CPB for 3 to 5 minutes but before cardioplegia. Once the crossclamp was applied to the aorta and the heart was arrested, PI was recorded at approximately 5, 20, 40, and 60 minutes after crossclamping.

For this exploratory study, a sample size of 13 subjects per group provided $80 \%$ power to detect a standardized effect size (ie, the difference in means divided by a common standard deviation) of 1.15 standard deviations using a 2-sided test having a significance level of .05. Two-sample $t$ tests were used to assess differences between pulsatile and nonpulsatile subjects for continuous variables, such as demographics and perfusion parameters. A $\chi^{2}$ test for linear trend was used to assess differences in pulsatile and nonpulsatile subjects with respect to the Jenkins risk category. A linear mixed effects model was fit to the perfusion index to compare the pulsatile and nonpulsatile subjects within each location (head or tube) at each time point. The linear mixed effects model is an extension of linear regression that accounts for the within-subject variability inherent in repeated measures designs. Inasmuch as there were two repeated factors in this model, location and time, the Kronecker product of two unstructured matrices was used in the mixed effects model to specify the variance-covariance matrix. Residual diagnostics were used to evaluate model fit. Inaasmuch as this is an exploratory study, no adjustments for multiple testing were done. All hypothesis tests were 2-sided and all analyses were performed with version 9.1 of the SAS System for Windows (SAS Institute, Inc, Cary, NC).

\section{RESULTS}

The patients receiving nonpulsatile perfusion were similar in age, weight, and body surface area to those receiving pulsatile perfusion. Inotropic support was comparable in both groups, but the mean arterial pressure in the pulsatile group 24 hours after CPB was higher than the nonpulsatile group. Urine output during the operation was also greater on pulsatile flow. Plasma free hemoglobin was comparable in both groups before and after CPB (Table 1).

Before the first incision and 3 to 5 minutes on $\mathrm{CPB}$, both groups had similar levels of MCA pulsatility. PI was greater in both the MCA and tubing throughout the duration of crossclamp time in the pulsatile group (Figure 1).

\section{DISCUSSION}

With FDA-approved CPB circuitry and components, pulsatility in the MCA was compared between pulsatile and nonpulsatile CPB. The minimal degree of pulsatility generated on nonpulsatile flow can be accounted for by the roller-type pump used in all cases. PI dampened significantly within aortic crossclamp duration in the nonpulsatile group. Under the assumption that pulsatility in the patient's MCA before the first incision is a representation of optimal cerebral perfusion, it is clear the pulsatile group achieved cerebral perfusion that closely approximates this physiologic environment.

TCD monitoring provides real-time information on cerebral perfusion during pediatric cardiac surgery. PI is a valid parameter for monitoring cerebral perfusion and will facilitate further research exploring the neurologic benefits of pulsatile flow in pediatric CPB. Over 300 patients are currently enrolled in such studies at the Penn State Children's Hospital.

Special thanks go to Mollie Barnes, CINM of Impulse Monitoring for data collection. 

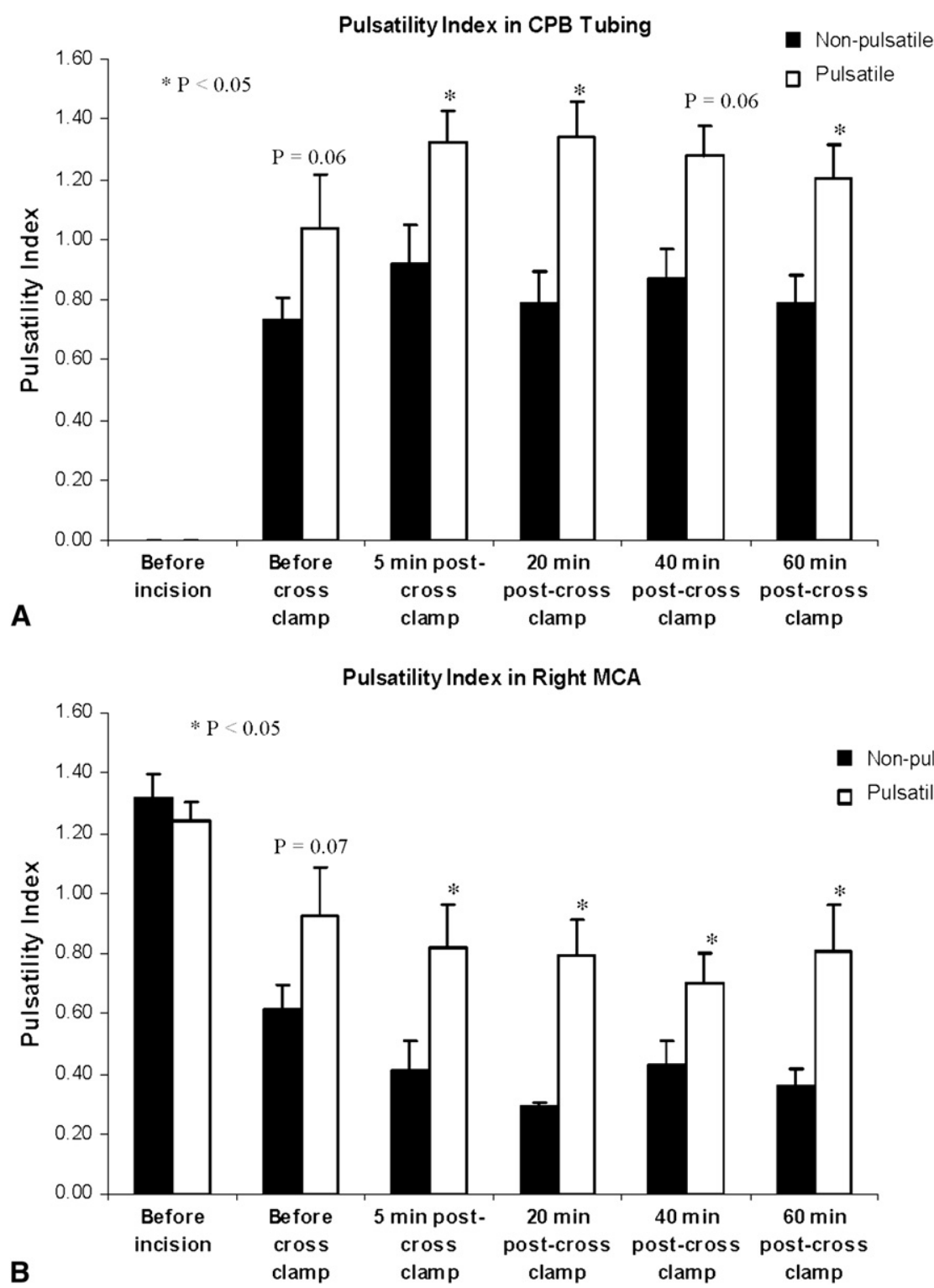

FIGURE 1. A, PI in CPB tubing. B, PI in the right MCA. PI, Pulsatility index; $C P B$, cardiopulmonary bypass; $M C A$, middle cerebral artery.

\section{References}

1. Polito A, Zaccaria R, Di Chiara L, Giorni C, Iacoella C, Sanders SP, et al. Cerebral blood flow during cardiopulmonary bypass in pediatric cardiac surgery: the role of transcranial Doppler - a systemic review of the literature. Cardiovasc Ultrasound. 2006;4:47.

2. Alkan T, Akcevin A, Ündar A, Türkoğlu H, Paker T, Aytaç A. Benefits of pulsatile perfusion on vital organ recovery during and after pediatric open heart surgery. ASAIO J. 2007;53:651-4.
3. Ündar A. Pulsatile versus nonpulsatile cardiopulmonary bypass procedures in neonates and infants: from bench to clinical practice [invited editorial]. ASAIO J. 2005;51:vi-x.

4. Reference guide: WinTCD software version 3.3. Madison (WI): Nicolet Biomedical Inc: 2002.

5. Ündar A, Eichstaedt HC, Masai T, Yang SQ, Bigley JE, McGarry MC, et al. Comparison of six pediatric cardiopulmonary bypass pumps during pulsatile and nonpulsatile perfusion. J Thorac Cardiovasc Surg. 2001;122:827-9. 